\title{
Strongly asymmetric square waves in a time-delayed system
}

\author{
Lionel Weicker, ${ }^{1}$ Thomas Erneux, ${ }^{1}$ Otti D'Huys, ${ }^{2}$ Jan Danckaert, ${ }^{2}$ Maxime Jacquot, ${ }^{3}$ Yanne Chembo, ${ }^{3}$ and Laurent Larger ${ }^{3}$ \\ ${ }^{1}$ Université Libre de Bruxelles, Optique Nonlinéaire Théorique, Campus Plaine, C.P. 231, 1050 Bruxelles, Belgium \\ ${ }^{2}$ Applied Physics Research Group (APHY), Vrije Universiteit Brussel, 1050 Brussel, Belgium \\ ${ }^{3}$ FEMTO-ST/Optics Department, UMR CNRS 6174, University of Franche-Comté, 16 Route de Gray, 25030 Besançon Cedex, France
}

(Received 16 May 2012; revised manuscript received 9 September 2012; published 29 November 2012)

\begin{abstract}
Time-delayed systems are known to exhibit symmetric square waves oscillating with a period close to twice the delay. Here, we show that strongly asymmetric square waves of a period close to one delay are possible. The plateau lengths can be tuned by changing a control parameter. The problem is investigated experimentally and numerically using a simple bandpass optoelectronic delay oscillator modeled by nonlinear delay integrodifferential equations. An asymptotic approximation of the square-wave periodic solution valid in the large delay limit allows an analytical description of its main properties (extrema and square pulse durations). A detailed numerical study of the bifurcation diagram indicates that the asymmetric square waves emerge from a Hopf bifurcation.
\end{abstract}

DOI: 10.1103/PhysRevE.86.055201

PACS number(s): 05.45.-a, 42.65.Sf, 02.30.Ks, 02.30.Oz

In the past three years, intensified efforts have been made to explore the physical effects of time delays [1-5]. In biology, time delays are called maturation periods, control reflexes, and cellular transport times, and they appear in various areas including neural networks, where propagation delays of the electrical signals connecting different neurons are taken into account [3]. In the industry, machine tool chatter is a persistent problem in metal cutting applications, where a delay term is typically involved in the applied force [6]. Optical feedback from a distant reflector is another example where a time delay is causing erratic intensity oscillations in lasers used in optical communication systems [7]. Time-delayed feedbacks are also used for practical applications. They might be deliberately implemented to control neural disturbances, e.g., to suppress undesired synchrony of firing neurons in Parkinson's disease [8]. They are also designed for producing new effects with optoelectronic oscillators (OEOs). An OEO typically incorporates a nonlinear (intensity) modulator, an optical fiber delay line, and an optical detector, followed by a bandpass filter, in a closed-loop resonating configuration. This hybrid microwave source is capable of generating, within the same optoelectronic cavity, either an ultralow-jitter (low-phase-noise) single tone microwave oscillation (narrow band filtering), as used in radar applications [9], or a wide band chaotic carrier (broadband filter) typically intended for physical encryption in high bit rate optical communications [10].

Because of the large variety of applications, there is an increasing interest for problems modeled by delay differential equations (DDEs) and their specific dynamical phenomena. A fundamental property of nonlinear dynamical systems controlled by a delayed feedback is their tendency to exhibit square-wave oscillations if the delay $\tau_{D}$ is sufficiently large. These oscillations typically consist of $2 \tau_{D}$-periodic transitions between two or more flat plateaus. The following scalar DDE,

$$
\varepsilon x^{\prime}=-x+f(x(s-1), \lambda),
$$

has been studied intensively for its square-wave solutions (the prime stands for the derivative with respect to the time $s$ ). Equation (1) arises in a variety of applications, for example, physiological control systems [11], the transmission of light through a ring cavity [12], and population biology [13]. Here the dimensionless time is $s \equiv t / \tau_{D}$ and $\varepsilon \equiv \tau / \tau_{D}>0$ is a small parameter defined as the ratio of the linear decay time $\tau$ to the delay $\tau_{D} . f(x, \lambda)$ denotes a nonlinear function of $x$, and $\lambda$ is a control parameter. Setting $\varepsilon$ to 0 reduces Eq. (1) to the following map relating $x_{n}=x(s)$ and $x_{n-1}=x(s-1)$ :

$$
x_{n}=f\left(x_{n-1}, \lambda\right) \text {. }
$$

The two fixed points of the period-2 solution of Eq. (2) are expected to approach each of the two plateaus of the squarewave solutions of Eq. (1) if $\varepsilon \rightarrow 0$. Significant contributions to the asymptotic relations between the solutions of the map (2) and the solutions of the DDE (1) have been made by Chow and Mallet-Paret [14], Mallet-Paret and Nussbaum [15], Chow et al. [16], and Hale and Huang [17]. Close to a Hopf bifurcation point $\lambda=\lambda_{0}(\varepsilon)$ of the DDE (1), the oscillations quickly change their shape from sinusoidal to square waves as the amplitude of the solution increases [18]. The square-wave oscillations then consist of sharp transition layers of a size proportional to $\varepsilon$, connecting the two plateaus, of lengths close to unity.

In this Rapid Communication, we address the question whether periodic square-wave oscillations of different plateau lengths are possible. These types of patterns may appear in systems modeled by Eq. (1) for particular functions $f(x, \lambda)$ and judicious choices of the initial conditions. But they are unstable for a long time [19] and have been called metastable [1]. Some of our recent experiments have revealed the stable occurrence of such asymmetric square waves using an optical system that is modeled by a second order DDE instead of Eq. (1). A particular feature of these square waves not anticipated by earlier investigations is the fact that they are nearly $\tau_{D}$ periodic. Figure 1 exhibits experimental stable $\tau_{D}$-periodic waves that were obtained from a broadband bandpass optoelectronic oscillator (OEO). The setup is depicted in the same figure and is a straightforward implementation of the broadband chaos generator described in Ref. [10]. It consists of an integrated optics Mach-Zehnder modulator (MZM) used to provide the nonlinear transformation as an electronically tunable two-wave interference. A dc bias can be adjusted externally to fix the zero drive interference condition (parameter $\Phi$ ). The MZM output is delayed in time by a 4-km fiber spool 

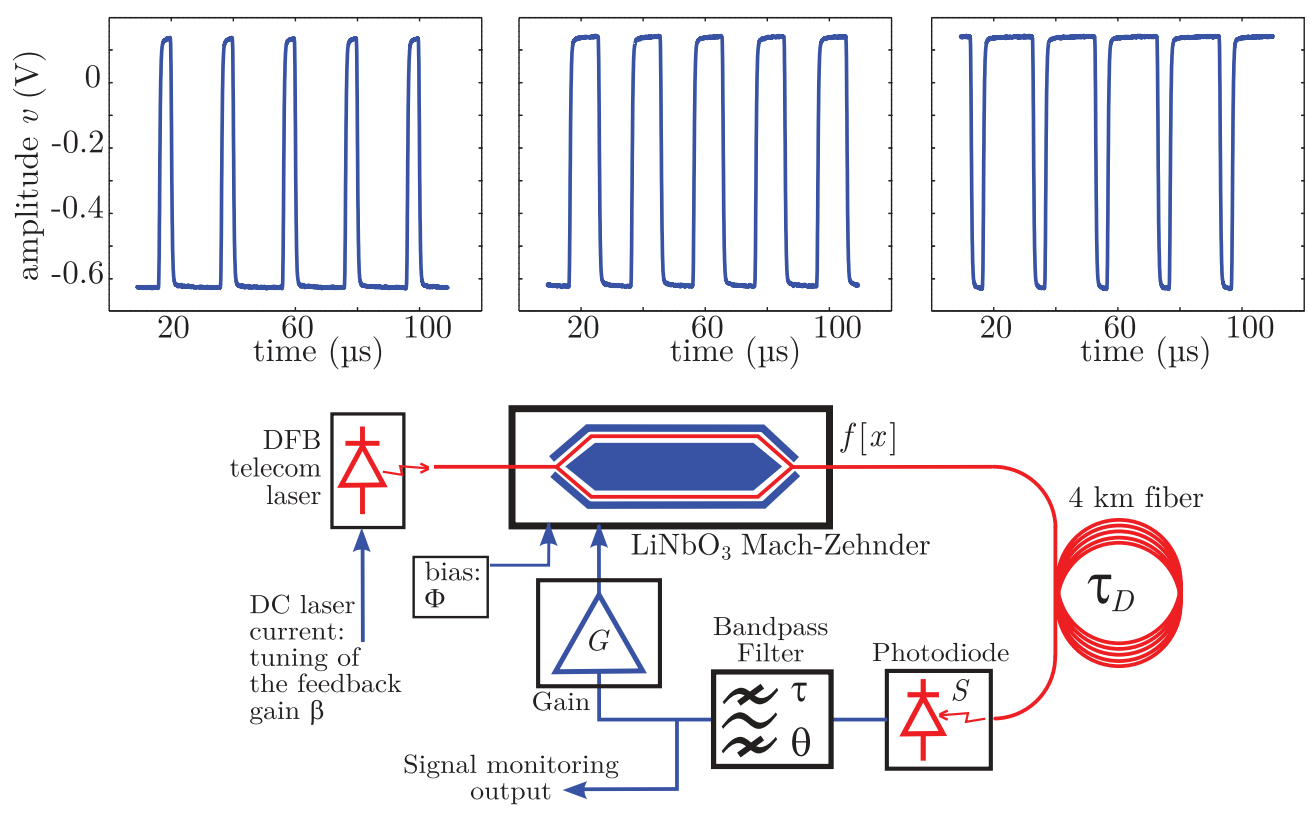

FIG. 1. (Color online) Upper: $\tau_{D}$-periodic square-wave oscillations for three values of the feedback phase $\phi$ increasing from left to right. The duty cycle is monotonically modified, but the period is unchanged. Lower: Experimental setup performing a bandpass electro-optic Ikeda dynamics.

( $\tau_{D} \simeq 20 \mu \mathrm{s}$ ), and then detected by a photodiode. A simple self-made electronic circuitry performs amplification as well as the dynamical limitation for the signal to be fed back on the MZM. The dynamics of the oscillation loop is thus controlled via its electronic Fourier filtering feature, which is forced to a broadband bandpass filter. The characteristic response time of the corresponding cascaded high pass and low pass first order filters are approximately $0.8 \mathrm{~s}(\theta)$ and $0.2 \mu \mathrm{s}$ $(\tau)$, respectively. The normalized gain $\beta$ of the oscillation loop is adjusted through the injection current of the cw $1.5-\mu \mathrm{m}$ distributed feedback (DFB) telecom semiconductor laser seeding optically the MZM. The plateau lengths of the waveform in Fig. 1 can be changed gradually by changing a control parameter ( $\Phi$ or $\beta$ ), but the overall pattern remains $\tau_{D}$ periodic. Numerical simulations of Eqs. (3) and (4) for long intervals of time reproduce these asymmetric waves but offer no clue to their nature. In order to have an analytical understanding of their properties, an asymptotic analysis of the OEO equations is proposed.

In dimensionless form, the evolution equations are given by (see Ref. [20] for details)

$$
\begin{gathered}
y^{\prime}=x, \\
\varepsilon x^{\prime}=-\delta y-x+\beta\left\{\cos ^{2}[x(s-1)+\Phi]-\cos ^{2} \Phi\right\},
\end{gathered}
$$

where $s=t / \tau_{D}$ is time measured in units of the delay. $\varepsilon=$ $\tau / \tau_{D}$ and $\delta=\tau_{D} / \theta$ are two small fixed parameters. The two control parameters are the feedback gain $\beta$ and the feedback offset phase $\Phi$. Under specific initial conditions and parameter values, Eqs. (3) and (4) admit square-wave solutions for $x$ with different plateau lengths (see Fig. 2). The periodic solution is nearly 1-periodic. We denote the length of the two plateaus by $s_{0}$ and $1-s_{0}$, respectively. $x_{01}$ and $x_{02}$ mark the extrema of $x$. The value of $\Phi$ is $-\pi / 4+0.1$. If $\Phi=-\pi / 4$, the square wave is perfectly symmetric $\left(s_{0}=1 / 2\right)$. Taking advantage of the small value of $\varepsilon$, we propose to construct a periodic solution satisfying the condition

$$
x(s-P)=x(s)
$$

where $P=1+2 \varepsilon r(\varepsilon)[r=O(1)]$. The leading approximation is obtained by setting $\varepsilon=0$ in Eqs. (3) and (4). The reduced equations with (5) and $P=1$ are

$$
\begin{gathered}
y^{\prime}=x \\
y=\delta^{-1}\left\{-x+\beta\left[\cos ^{2}(x+\Phi)-\cos ^{2} \Phi\right]\right\} .
\end{gathered}
$$

The function (7) is represented in Fig. 2(b) and exhibits three branches provided that $\beta>1$. The evolution of $x$ and $y$ along the left and right branches can be determined analytically by inserting (7) into (6) and solving the resulting first order equation in $x$. However, simple expressions of the solution
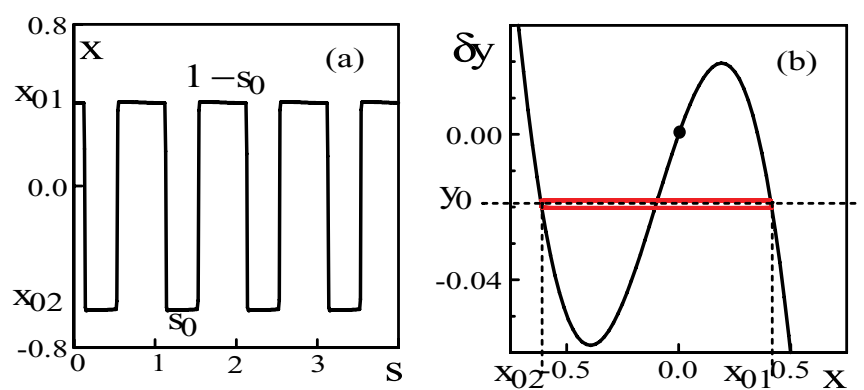

FIG. 2. (Color online) (a) Long time periodic solution obtained numerically from Eqs. (3) and (4). $\varepsilon=10^{-3}, \delta=8.43 \times 10^{-3}$, $\Phi=-\pi / 4+0.1, \beta=1.2$; the initial conditions are $x=-1(-1 \leqslant$ $s<-1 / 3), x=1(-1 / 3 \leqslant s<0)$, and $y(0)=0$. (b) The periodic solution is represented in red (gray) in the phase plane $(x, y)$. The black line is the function (7), and the black dot is the unique steady state $(x, y)=(0,0)$. 
can be obtained by taking advantage of the small value of $\delta$. Specifically, we seek a solution of the form

$$
\begin{gathered}
y=\delta^{-1} y_{0}+y_{1 j}(s)+\cdots, \\
x=x_{0 j}+\delta x_{1 j}(s)+\cdots,
\end{gathered}
$$

where $j=1$ or 2 refers to the time domains $0<s<s_{0}$ and $s_{0}<s<1$, respectively [see Fig. 2(a)]. The leading order problem as $\delta \rightarrow 0$ is

$$
\begin{gathered}
y_{0}^{\prime}=0, \\
-x_{0 j}-y_{0}+\beta\left[\cos ^{2}\left(x_{0 j}+\Phi\right)-\cos ^{2} \Phi\right]=0 .
\end{gathered}
$$

We already know that for a finite interval of $y_{0}$, Eq. (11) admits more than one solution [see Fig. 2(b)]. The solutions corresponding to the left and right branches are denoted by $x_{02}<0$ and $x_{01}>0$, respectively. Their values are unknown because $y_{0}$ is still unknown. This motivates the analysis of the next problem for $y_{1 j}$ and $x_{1 j}$, given by

$$
\begin{gathered}
y_{1 j}^{\prime}=x_{0 j}, \\
-x_{1 j}-y_{1 j}-\beta \sin \left(2 x_{0 j}+2 \Phi\right) x_{1 j}=0,
\end{gathered}
$$

where $j=1$ or 2 . We solve Eq. (12) with the initial conditions $y_{11}(0)=0$ and $y_{12}\left(s_{0}\right)=y_{11}\left(s_{0}\right)$. We then determine $x_{11}$ and $x_{12}$ using Eq. (13). We obtain

$$
\begin{gathered}
y_{11}=x_{01} s, \\
y_{12}=x_{01} s_{0}+x_{02}\left(s-s_{0}\right), \\
x_{11}=-\frac{x_{01} s}{1+\beta \sin \left(2 x_{01}+2 \Phi\right)}, \\
x_{12}=-\frac{\left[x_{01} s_{0}+x_{02}\left(s-s_{0}\right)\right]}{1+\beta \sin \left(2 x_{02}+2 \Phi\right)} .
\end{gathered}
$$

The 1-periodicity condition requires that $y_{12}(1)=0$, and from (15) we obtain an equation relating the unknowns $x_{01}, x_{02}$, and $s_{0}$ :

$$
x_{01} s_{0}+x_{02}\left(1-s_{0}\right)=0 .
$$

Moreover, continuity of $x_{11}$ and $x_{12}$ at $s=s_{0}$ requires

$$
\cos \left(x_{01}+x_{02}+2 \Phi\right) \sin \left(x_{01}-x_{02}\right)=0 .
$$

This is a first condition for the unknowns $x_{01}$ and $x_{02}$. Because $x_{01} \neq x_{02}$, the solution of Eq. (19) is $x_{01}+x_{02}+2 \Phi=$ $\pm \pi / 2+2 n \pi$. The numerical solution in Fig. 2 verifies the condition

$$
x_{01}+x_{02}+2 \Phi=-\pi / 2 .
$$

We consider Eq. (11). Subtracting the equation with $j=1$ and the equation with $j=2$ gives

$$
-\left(x_{01}-x_{02}\right)-\beta \sin \left(x_{01}+x_{02}+2 \Phi\right) \sin \left(x_{01}-x_{02}\right)=0 \text {. }
$$

Using (20) then allows to eliminate $x_{02}$ in (21):

$$
-\left(2 x_{01}+2 \Phi+\pi / 2\right)+\beta \sin \left(2 x_{01}+2 \Phi+\pi / 2\right)=0 .
$$

This provides $x_{01}=x_{01}(\beta)$, which can be determined in the implicit form $\beta=\beta\left(x_{01}\right)$. The values of $x_{02}$ and $s_{0}$ are then

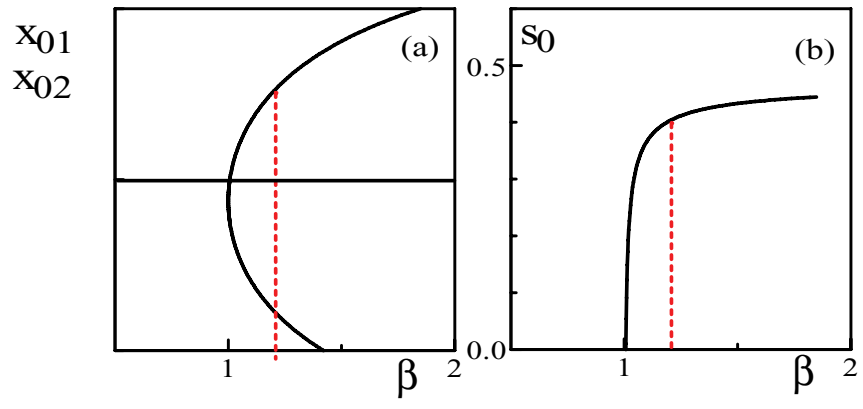

FIG. 3. (Color online) (a) and (b) Analytical bifurcation diagram for the extrema of $x\left(x_{01}\right.$ and $\left.x_{02}\right)$ and the length of the first plateau $\left(s_{0}\right)$. The vertical dashed lines mark the value of $\beta=1.2$ used in the simulations in Fig. 2(a). The predicted values of the extrema and the length of the first plateau are in excellent quantitative agreement with the values documented from the numerical simulations.

found by using (20) and (18) (see Fig. 3). Furthermore, using (22) in (11) with $j=1$, one gets

$$
y_{0}=\Phi+\frac{\pi}{4}-\frac{\beta}{2} \cos (2 \Phi)
$$

Equation (11) admits a third root $x_{03}\left(x_{02}<x_{03}<x_{01}\right)$, which can be verified from (11) and (23) as being $x_{03}=-(\Phi+\pi / 4)$. Combining this value with (20) leads to the root symmetry property,

$$
z_{0}=x_{01}-x_{03}=x_{03}-x_{02},
$$

which will play a crucial role for the transition layers (see below).

In summary, we concentrated on the branch of strongly asymmetric square waves. As $\beta$ progressively decreases from a value $\beta$ larger than one, we note from Figs. 3(a) and 3(b) that both the extrema of the square wave and the plateau length $s_{0}$ decrease to zero as $\beta \rightarrow 1$.

In order to complete our asymptotic analysis, we need to investigate the fast transition layers between $x_{01}$ and $x_{02}$. To this end, we introduce the transition layer variable $\zeta_{1} \equiv s / \varepsilon$ (jump down) or $\zeta_{2} \equiv\left(s-s_{0}-\varepsilon r\right) / \varepsilon$ (jump up) into Eqs. (3) and (4). The correction term $\varepsilon r$ represents the contribution to the period of each transition layer $(P=1+2 \varepsilon r)$ and $r$ needs to be determined from the transition layer equations. From the leading order equations as $\varepsilon \rightarrow 0$, we find that $\delta y=y_{0}$, where $y_{0}$ is defined by (23) and a first order DDE for $x$. Because of (24), we note that $z\left(\zeta_{2}\right)=-z\left(\zeta_{1}\right)$ where $z \equiv x-x_{03}$. We then find that the transition layer problems are described by the same equation for $z$ and only differ by their boundary conditions. For the jump down transition, we find

$$
z^{\prime}=-z+\frac{\beta}{2} \sin \left[2 z\left(\zeta_{1}+2 r\right)\right]
$$

where the prime means differentiation with respect to $\zeta_{1}$. The boundary conditions are $z( \pm \infty)=\mp z_{0}$. Equation (25) is covered by the general theory of Hale and co-workers $[14,17$, 21]. Specifically, we seek a heteroclinic orbit for some value of $r$ that is a trajectory joining $z_{0}$ and $-z_{0}$ at times $\zeta=\mp \infty$. In the limit, $\beta \rightarrow 1$ we find that $r \rightarrow 1 / 2$. For an arbitrary value of $\beta$, the transition layer equation needs to be solved numerically. 

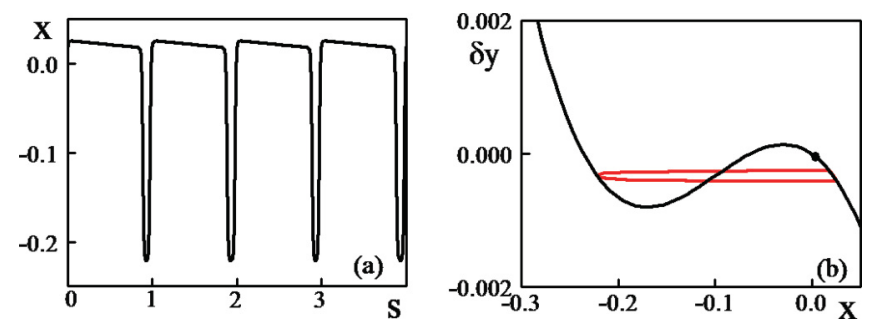

FIG. 4. (Color online) Same values of the fixed parameters and initial conditions as in Fig. 2 except $\beta=1.01$. (a) Numerical square-wave solution exhibiting a small plateau length $s_{0}$. (b) Periodic solution in the phase plane. The dot marks the steady state $(x, y)=$ $(0,0)$.

Close to $\beta=1$, the asymptotic analysis assuming $s_{0}=$ $O(1)$ and fast transition layers of size $\varepsilon$ is no longer valid. The smallest value of $\beta$ where we numerically found the square wave is close to $\beta=\beta_{c} \simeq 1.01$ (see Fig. 4). Note that the maxima of $x$ are close to the steady state value $x=0$. As we further decrease $\beta$, we find $x=0$ as the only stable solution. A systematic study of the bifurcation diagram by integrating numerically Eqs. (3) and (4) and by using a continuation method (DDE-BIFTOOL [22]) indicates that the asymmetric square-wave solutions belong to a Hopf bifurcation branch that emerges from $x=0$ at $\beta=1.020$ with a period close to 1 . It is the first Hopf bifurcation from the basic steady state $x=0$. The branch of periodic solutions is subcritical and unstable but quickly folds back to stable asymmetric square waves, as seen in Fig. 4.

The asymmetric square-wave regime is not the only stable periodic solution of Eqs. (3) and (4). Although the number of primary Hopf bifurcation points increase as $\beta$ is increased from $\beta=1.020$, we found that only two bifurcations lead to stable branches: the branch of asymmetric square-wave solutions studied in this Rapid Communication and a branch of low frequency oscillations that emerges from a Hopf bifurcation point at $\beta=1.025$ with a period close to $70\left(\simeq \delta^{-1}\right.$, related physically to $\theta$, as already found in Ref. [20]). These two branches dominate the bifurcation diagram as we gradually increase $\beta$ from 1 or decrease $\beta$ from a large value. No other stable time-dependent regimes have been found in the interval $0<\beta<2$. Details on the analysis of the transition layers and on the numerical bifurcation diagrams will be published elsewhere.

A similar asymmetric square wave has been explored recently both experimentally and numerically for an OEO operating under quite different parameter values [23]. We are currently looking into whether a similar Hopf bifurcation mechanism is responsible for these oscillations.

T.E. acknowledges the support of the F.N.R.S. (Belgium). L.W. acknowledges the Belgian F.R.I.A. for support through a Ph.D. scholarship. This work was supported by the European project PHOCUS (FP7 Grant No. 240763). L.L. thanks the support of the Institut Universitaire de France.
[1] T. Erneux, Applied Delay Differential Equations (Springer, Berlin, 2009).

[2] Complex Time-Delay Systems, edited by F. M. Atay (Springer, Berlin, 2010).

[3] W. Just, A. Pelster, M. Schanz, and E. Schöll, Philos. Trans. R. Soc. London, Ser. A 368, 303 (2010).

[4] J. Vib. Control 16 (7-8) (2010), special issue on time delay systems, edited by T. Kalmár-Nagy, N. Olgac, and G. Stépán.

[5] H. Smith, An Introduction to Delay Differential Equations with Applications to the Life Sciences (Texts in Applied Mathematics) (Springer, Berlin, 2010).

[6] G. Stépan, in Dynamics and Chaos in Manufacturing Processes, edited by F. C. Moon (Wiley, New York, 1998), pp. 165-192.

[7] Unlocking Dynamical Diversity: Optical Feedback Effects on Semiconductor Lasers, edited by D. M. Kane and K. A. Shore (Wiley, New York, 2005).

[8] M. Rosenblum and A. Pikovsky, Phys. Rev. E 70, 041904 (2004).

[9] J. Lasri, P. Devgan, R. Tang, and P. Kumar, Opt. Express 11, 1430 (2003); IEEE Photonics Technol. Lett. 16, 263 (2004).

[10] L. Larger and J.-P. Goedgebuer, C. R. Phys. 5, 609 (2004).

[11] M. C. Mackey and L. Glass, Science 197, 287 (1977).

[12] K. Ikeda, Opt. Commun. 30, 257 (1979).
[13] W. S. C. Gurney, S. P. Blythe, and R. M. Nisbet, Nature (London) 287, 17 (1980).

[14] S. N. Chow and J. Mallet-Paret, in Coupled Nonlinear Oscillators, Proceedings, Los Alamos, New Mexico, 1981, edited by J. Chandra and A. C. Scott (North-Holland Publ. Co., Amsterdam-New York, 1983).

[15] J. Mallet-Paret and R. D. Nussbaum, Ann. Mat. Pura Appl. 145, 33 (1986).

[16] S. N. Chow, J. K. Hale, and W. Huang, Proc. R. Soc. Edinburgh, Sect. A: Math. 120, 223 (1992).

[17] J. K. Hale and W. Z. Huang, J. Differ. Equ. 114, 1 (1994); Z. Angew. Math. Phys. 47(1), 57 (1996).

[18] T. Erneux, L. Larger, M. W. Lee, and J. P. Goedgebuer, Physica D 194, 49 (2004).

[19] A. N. Sharkovsky, Yu.L. Maistrenko, and E. Yu. Romanenko, Difference Equations and Their Applications (Kluwer Academic, Dordrecht, 1993).

[20] M. Peil, M. Jacquot, Y. K. Chembo, L. Larger, and T. Erneux, Phys. Rev. E 79, 026208 (2009).

[21] W. Huang, J. Differ. Equ. 168, 239269 (2000).

[22] K. Engelborghs, T. Luzyanina, and D. Roose, ACM Trans. Math. Software 28, 1 (2002)

[23] D. P. Rosin, K. E. Callan, D. J. Gauthier, and E. Schöll, Europhys. Lett. 96, 34001 (2011). 